\title{
A Note on Lange on Contingent Necessity-Makers
}

\author{
Nathan Wildman ${ }^{1}$ (D)
}

Received: 4 June 2018 / Accepted: 2 May 2019 / Published online: 29 May 2019

(c) The Author(s) 2019

\begin{abstract}
Lange (Analysis 68:120-128, 2008) has argued that contingencies lack the modal strength to be necessity-makers. Here, I argue that Lange's case turns upon a faulty premise, and that there is no obvious fixes he might pursue. The general upshot is that his argument gives us no reason to think that contingencies could not be necessity-makers after all.
\end{abstract}

Blackburn (1993 [1986]) poses a dilemma for any realist attempt to explain why some fact A is necessary. ${ }^{1}$ Suppose that we explain some fact A's necessity (i.e., we explain why A has the modal status of being necessary) by appeal to some fact $\mathrm{F}$. This fact $\mathrm{F}$ must itself be either necessary or contingent. If $\mathrm{F}$ is necessary, then, says Blackburn, there 'will be a bad residual 'must' (1993: 54)—we will have failed to properly explain what A's necessity consists in, as F is the same kind of fact as what we are meant to be spelling out. Meanwhile, if $\mathrm{F}$ is contingent, then there is 'strong pressure to feel that the original necessity has not been explained or identified, so much as undermined.' So, either the explanans F 'shares the modal status of the original, and leaves us dissatisfied, or it does not, and leaves us equally dissatisfied' (Blackburn 1993: 54).

Blackburn intended this dilemma to motivate rejecting a realist understanding of modality; in its place, he advocated a quasi-realist view. However, most metaphysicians have been hesitant to give up on realism about modality so quickly. Consequently, since Blackburn's initial presentation, a cottage industry has developed, centred upon refining and objecting to the arguments underpinning the dilemma's two horns. ${ }^{2}$

\footnotetext{
1 Following Lange (2008: 120fn1), I take the dilemma and the subsequent discussion to exclusively concern de dicto, alethic necessities. Consequently, I hereby ignore e.g. doxastic and epistemic necessities, as well as de re modal claims.

2 See e.g. Hale (2002, 2013), Hanks (2008), Cameron (2010), Morato (2014) and Wildman (2017).
}

Nathan Wildman

n.w.wildman@uvt.nl

1 TiLPS/Department of Philosophy, Tilburg University, Tilburg, The Netherlands 
Recently, Lange has offered a refinement of the contingency horn, arguing that contingencies 'lack the modal strength to be necessity-makers' (2008: 122). ${ }^{3}$ This paper disputes Lange's conclusion. It does so by demonstrating that one of the argument's central premises is subject to a range of counter-examples. Further, neither of the two most plausible responses Lange might pursue in the face of these counter-examples are viable, as they either invalidate the overall argument or stand at odds with the reasoning undergirding the argument's premises. The end result is that Lange gives us no reason to think that contingencies could not be necessity-makers after all.

Before I proceed, a quick point about notation: following Lange (p. 122), I use capital letters (A, C, F, etc.) to represent hypothetical states of affairs that obtain (or not) in a given world purely in virtue of how things are in that world. So, for example, 'C $\square \rightarrow$ A' cannot be interpreted as 'Had I been 120 kilos, I would have been 20 kilos heavier than I actually am', since the latter fact is a matter of how things are not just in that possible world, but also in the actual world.

\section{Lange Against Contingent Necessity-Makers}

Lange's argument relies upon three major premises. The first is:

Circumstantial Immunity $\square \mathrm{A}$ iff for any $\mathrm{C}$ and $\mathrm{C}^{\prime}$ where $\nabla_{\mathrm{C}}$ and $\nabla_{\mathrm{C}^{\prime}, \mathrm{C}} \square \rightarrow \mathrm{A}$ and $\mathrm{C}^{\prime} \square \rightarrow(\mathrm{C} \square \rightarrow \mathrm{A})^{4,5}$

The underlying thought is that necessity is truth come what may. So, just as something that must be the case obtains regardless of whatever else happens, something that happens regardless of what else obtains must be the case.

According to Lange, this tight relation between A's necessity and the various counterfactuals highlighted in CI suggests that 'if $\mathrm{F}$ is A's necessity-maker, then $\mathrm{F}$ is responsible for the truth of the various counterfactuals that $[\mathbf{C I}]$ associates with A's necessity' (pp. 123-124). However, as he admits, this isn't entirely correct. Suppose $A$ is necessary in virtue of $F$. One of the counterfactuals associated with A's necessity is that A would have been true had $A$ and $B$ been true-i.e., $((A \wedge B) \square \rightarrow A)$. But what's responsible for the truth of this counterfactual is that A entails $\mathrm{A}$, not $\mathrm{F}$.

However, even in these cases, $\mathrm{F}$ is still responsible for some of the non-trivial nested counterfactuals $\mathbf{C I}$ associates with A. This leads Lange to posit his second assumption:

\footnotetext{
3 All references are to Lange (2008) unless otherwise specified.

4 This is Lange's premise (4). Further, I assume that this and the following principles are universal, holding for all relevant $\mathrm{A}, \mathrm{C}$, and F's.

5 Notably, Lewis (1973) rejects CI for natural necessity, because, according to him, the closest C-worlds are worlds where C obtains due to small 'miracles', and hence aren't A-worlds. Additionally, CI is neutral on whether counterfactuals are metaphysically or epistemically prior to necessities, or vice versa (c.f. e.g. Williamson 2007: Chapter 5, Kment 2006).
} 
Counterfactual Responsibility If $\mathrm{F}$ is responsible for $\square \mathrm{A}$, then, for any $\mathrm{C}$ where $\nabla_{\mathrm{C}}$, there is either some truth $(\mathrm{C} \square \rightarrow \mathrm{A})$, or some truth $\mathrm{C} \square \rightarrow\left(\mathrm{C}^{\prime} \square \rightarrow \mathrm{A}\right)$ for some $\mathrm{C}^{\prime}$ where $\vee \mathrm{C}^{\prime}$, for which $\mathrm{F}$ is responsible ${ }^{6}$

Finally, Lange's third assumption is

Counterfactual Entailment If $\mathrm{F}$ is responsible for $\mathrm{C} \square \rightarrow \mathrm{A}$ [or for $\mathrm{C} \square \rightarrow\left(\mathrm{C}^{\prime} \square \rightarrow\right.$ A)], then $\mathrm{C} \square \rightarrow \mathrm{F}^{7}$

He offers two, related lines of motivation for CE. The first takes it as a generalization from three test cases. It's true that, had match $m$ been struck, it would have lit because $m$ is dry and oxygenated (and various relevant laws of nature hold). But, according to Lange, this explanation only succeeds if the match would have still been dry and oxygenated had it been struck-otherwise, that the match is dry and oxygenated is 'irrelevant to what would have happened, had the match been struck' (pp. 124-125). Similarly, that an electric field's strength at a given spatiotemporal location $l$ is 1 dyne per statcoulomb is responsible for the truth of the counterfactual that, had $l$ been occupied by an entity $b$ with 5 statcoulomb charge, $b$ would have experienced a 5 dyne electric force. According to Lange, this explanation presupposes that, had $b$ been present in $l$, the field's strength wouldn't have been different. Finally, take a causal pre-emption case: Billy and Suzy are (accurately) throwing rocks at a bottle, and Suzy's rock hits first, shattering it. Because of Billy's rock, the bottle still would have shattered had Suzy not thrown her rock. This explanation requires that Billy would have thrown his rock had Suzy not thrown hers-otherwise, nothing ensures that the bottle would shatter. In each case, we've counterfactuals of the form $\mathrm{C} \square \rightarrow \mathrm{A}$ which obtain because of some fact $\mathrm{F}$ and, had $\mathrm{C}$ obtained, $\mathrm{F}$ would have too-just like CE says.

The second line of motivation is that $\mathbf{C E}$ follows from two other, minor assumptions:

Transitive Responsibility If $\mathrm{F}$ is responsible for $\mathrm{C} \square \rightarrow \mathrm{A}$, then, had $\mathrm{C}$ obtained, $\mathrm{F}$ would have been responsible for $\mathrm{A}$

Explanans Factivity In any possible world: $\mathrm{F}$ is responsible for A only if $\mathrm{F}$

And while EF straightforwardly follows from the standard assumption that explanatory relations are factive, $\mathbf{T R}$ is 'non-trivial'. However, it is

borne out in our examples. For instance, in the closest possible world where a 5 statcoulomb charge is present at the given location, its experiencing a 5 dyne electric force there is accounted for by the electric field strength there being 1 dyne per statcoulomb (p. 125).

\footnotetext{
6 This is Lange's (5).

7 The following are Lange's (6), (7), and (8) respectively.
} 
Hence the two lines of support for $\mathbf{C E}$ converge, both turning upon generalizing from the three examples, a point to which we'll return shortly.

With these three assumptions in place, we can run Lange's argument: Assume F is responsible for $\square \mathrm{A}$. By $\mathbf{C R}$, it follows that, for any possible $\mathrm{C}$, there is some truth of the form $(\mathrm{C} \square \rightarrow \mathrm{A})$ for which $\mathrm{F}$ is responsible. Hence, by $\mathbf{C E}$, for any possible $\mathrm{C}$, $\mathrm{C} \square \rightarrow \mathrm{F}$. Finally, because $\mathrm{F}$ has been shown to satisfy its right-hand-side, applying $\mathbf{C I}$ entails that $\mathrm{F}$ is necessary. As this means $\mathrm{F}$ can be responsible for A's necessity only if $\mathrm{F}$ is also necessary, Lange concludes there can be no contingent necessity-makers.

\section{Against CE and TR}

The problem is that one of the argument's central premises-namely, $\mathbf{C E}$ - and one of the minor premises intended to support $\mathbf{C E}$ - namely, $\mathbf{T R}$ - are subject to counter-example.

Suppose magnet $m$ has a positively-charged end $p$ and a negatively charged end $n$. Because $p$ is positively charged, were $m$ to undergo magnetic reversal, $p$ would be negatively charged. Consequently, it's the case that, that $p$ is positively charged is responsible for the truth of the counterfactual that $p$ would be negatively charged were $m$ to undergo magnetic reversal. But since $p$ 's being negatively charged entails it's not positively charged, it's not the case that, were $m$ to undergo magnetic reversal, $p$ would be positively charged.

With this in mind, let $\mathrm{F}$ be that $p$ is positively charged, $\mathrm{C}$ be that $m$ undergoes magnetic reversal, and $\mathrm{A}$ be that $p$ is negatively charged. In this case, $\mathrm{F}$ is responsible for $\mathrm{C} \square \rightarrow \mathrm{A}$, but, were $m$ to undergo magnetic reversal, then it's not the case that $p$ is positively charged-i.e., $\mathrm{C} \square \rightarrow \neg \mathrm{F}$. And, of course, if $\mathrm{C} \square \rightarrow \neg \mathrm{F}$, then $\neg(\mathrm{C} \square \rightarrow \mathrm{F})$. Consequently, $\mathbf{C E}$ is false in this case. Further, we know that, were $m$ to undergo magnetic reversal, then $p$ would no longer be positively charged. By EF, it therefore follows that, were $m$ to be magnetically reversed, $p$ 's being positively charged couldn't be responsible for anything. So, in such a circumstance, $p$ 's being positively charged wouldn't be responsible for $p$ 's being negatively charged! Hence we've a counter-example to TR: an instance where $\mathrm{F}$ is responsible for $\mathrm{C} \square \rightarrow \mathrm{A}$, but where, given C's obtaining, $\mathrm{F}$ isn't responsible for $\mathrm{A} .^{8}$

More generally, if $\mathrm{F}$ is some contingent fact with at least one complement fact $\mathrm{A}-$ i.e., a contingent fact the obtaining of which entails that $\neg \mathrm{F}$, and $\mathrm{C}$ is the obtaining of some operation that 'flips' $\mathrm{F}$ to $\mathrm{A}$, we have a counter-example to $\mathbf{C E}$ and TR. In all these 'flipping' cases, $\mathrm{F}$ is responsible for the truth of $\mathrm{C} \square \rightarrow \mathrm{A}$, but, because $\mathrm{A}$ and $\mathrm{F}$ are incompatible, it's not the case that $\mathrm{C} \square \rightarrow \mathrm{F}$. For example, let $\mathrm{F}$ be that the paint in this bucket is pure red, $\mathrm{C}$ that some white paint is added into the bucket,

\footnotetext{
${ }^{8}$ Objection: Lange could save TR by appealing to the fact that, had $m$ undergone magnetic reversal, $p$ 's *actually* being positively charged would be responsible for $p$ 's being negatively charged. Reply: This violates the restriction, set by Lange, that the relevant states of affairs are ones that obtain (or not) in a given world purely in virtue of how things are in that world.
} 
and A that the paint in the bucket is pink. In this case, the paint's being pure red is responsible for the truth of 'were white paint to be added, the paint in the bucket would be pink', but it not the case that were white paint to be added, the paint in bucket would be pure red, nor that the paint's being pure red is responsible for the paint's being pink.

\section{First Response: Restricting the Principles}

There are two lines of response Lange might pursue. The first involves modifying CE and/or TR so as to avoid the counterexamples. One way Lange might try to do so is by restricting the relevant principles to compossible F's and A's:

CE* $^{*}$ If (F is responsible for $\mathrm{C} \square \rightarrow \mathrm{A}$ [or for $\left.\mathrm{C} \square \rightarrow\left(\mathrm{C}^{\prime} \square \rightarrow \mathrm{A}\right)\right]$ ) and $\diamond(\mathrm{F}$ and $\mathrm{A})$, then $\mathrm{C} \square \rightarrow \mathrm{F}$

TR* If ( $F$ is responsible for $C \square \rightarrow A$ ) and $\diamond(F$ and $A$ ), then had $C$ obtained, $F$ would have been responsible for $\mathrm{A}$

These restrictions block 'flipping' counter-examples but face new ones. Specifically, we will have a new counter-example whenever we have an A that is multiply realizable and compossible with $\mathrm{F}$, and a $\mathrm{C}$ that 'transforms' $\mathrm{F}$ to $\mathrm{A}$ in such a manner as to entail that $\mathrm{F}$ is not the case (e.g. because $\mathrm{C}$ involves a change in a relevant object's properties or circumstances). For example, let $\mathrm{F}$ be that the paint in this bucket is pure red, $\mathrm{C}$ be that some blue paint is mixed into the bucket and the paint is examined through a blue filter, and A that the paint in the bucket appears red. That the paint is pure red is responsible for the truth of the counterfactual, and the paint's being pure red and appearing pure red are certainly compossible, but it's not the case that, were blue paint added and the paint examined through a blue filter, the paint would be pure red (rather, it'd be violet). ${ }^{9}$ So $\mathbf{C E}^{*}$ is just as flawed as $\mathbf{C E}$ : while restricting to compossible cases blocks 'flipping' counterexamples, the door is still open for 'transformation' ones.

Pushing further, Lange might restrict to mutually compossible F's, C's, and A's:

CE+ If ( $F$ is responsible for $C \square \rightarrow A$ [or for $\left.C \square \rightarrow\left(C^{\prime} \square \rightarrow A\right)\right]$ ) and $\diamond(F$ and $C$ and A), then $\mathrm{C} \square \rightarrow \mathrm{F}$

TR+ If ( $\mathrm{F}$ is responsible for $\mathrm{C} \square \rightarrow \mathrm{A})$ and $\diamond(\mathrm{F}$ and $\mathrm{C}$ and $\mathrm{A})$, then had $\mathrm{C}$ obtained, $\mathrm{F}$ would have been responsible for $\mathrm{A}$

Yet replacing $\mathbf{C E}$ with $\mathbf{C E}+$ renders Lange's argument invalid. Assume $\mathrm{F}$ is responsible for $\square$ A. By $\mathbf{C R}$, it follows that, for any possible $\mathrm{C}$, there is some truth of the form $(\mathrm{C} \square \rightarrow \mathrm{A})$ for which $\mathrm{F}$ is responsible. But nothing assumed so far guarantees

\footnotetext{
9 This case also works as a counterexample to the embedded counterfactual reading: let $\mathrm{F}$ be that the paint in the bucket is red, $\mathrm{C}$ be that blue paint is mixed into the bucket, $\mathrm{C}$ ' be that the paint is examined through a blue filter, and $\mathrm{A}$ be that the paint appears red.
} 
that $\mathrm{C}, \mathrm{F}$, and $\mathrm{A}$ are all jointly compossible, meaning the antecedent of $\mathbf{C E}+$ isn't satisfied. To get this, Lange must add as an additional assumption that $\mathrm{C}$ is compossible with F. ${ }^{10}$ But once we do so, it's not the case that, for all possible C's, $\mathrm{C} \square \rightarrow \mathrm{F}-$ instead, it's only for all compossible-with-F C's, $\mathrm{C} \square \rightarrow \mathrm{F}$. And unless we assume that $\mathrm{F}$ is necessary-which begs the question-the compossible-with-F-circumstances are only a proper subset of all possible circumstances. ${ }^{11}$ This means there's no way to satisfy the right-hand-side of $\mathbf{C I}$ and complete the argument. Consequently, were Lange to employ $\mathbf{C E}+$, his argument simply breaks down.

Alternatively, Lange might appeal to the times that F, C, and A occur at. ${ }^{12}$ For example, in the magnet case, we intuitively first have p's being positively charged, then the reversal, and then p's being negatively charged. Incorporating this temporal progression, Lange might modify CE/TR to:

CE-T If (F is responsible for $\mathrm{C} \square \rightarrow \mathrm{A}$ [or for $\left.\mathrm{C} \square \rightarrow\left(\mathrm{C}^{\prime} \square \rightarrow \mathrm{A}\right)\right]$ ) and (F occurs earlier than $\mathrm{A})$, then $\mathrm{C} \square \rightarrow \mathrm{F}$

TR-T If ( $F$ is responsible for $C \square \rightarrow A$ ) and ( $F$ occurs earlier than $A$ ), then, had $C$ obtained, $\mathrm{F}$ would have been responsible for $\mathrm{A}$

However, replacing CE with CE-T undermines the argument. Assume F is responsible for $\square$ A. By $\mathbf{C R}$, it follows that, for any possible $\mathrm{C}$, there is some truth of the form $(\mathrm{C} \square \rightarrow \mathrm{A})$ for which $\mathrm{F}$ is responsible. But, if $\mathrm{A}$ is necessary, then it plausibly occurs at every time, including times before (and at which) F occurs. Consequently, it is not the case that F occurs earlier than A. Hence the antecedent of CE-T isn't satisfied, and the argument grinds to a halt.

Finally, Lange might note that, in the counterexamples, the A to be explained is always contingent. However, in the present context, we are really only interested in explaining necessities - that is, cases where A is necessary. With this in mind, Lange could restrict $\mathbf{C E} / \mathbf{T R}$ in the following manner ${ }^{13}$ :

CE-N If $\mathrm{F}$ is responsible for $\mathrm{C} \square \rightarrow \mathrm{A}$ [or for $\mathrm{C} \square \rightarrow\left(\mathrm{C}^{\prime} \square \rightarrow \mathrm{A}\right)$ ] and $\square \mathrm{A}$, then $\mathrm{C} \square \rightarrow \mathrm{F}$ TR-N If ( $F$ is responsible for $C \square \rightarrow A$ ) and $\square \mathrm{A}$, then, had $\mathrm{C}$ obtained, $\mathrm{F}$ would have been responsible for $\mathrm{A}$

However, these do not help. For one, both fail in cases where we have an $\mathrm{F}$ that is both contingent and responsible for $\square \mathrm{A}$, an A that is necessary, and a (possibly occurring) $C$ such that C's occurring entails $\neg$ F. In such a case, it follows by $\mathbf{C R}$ that $\mathrm{F}$ is responsible for $(\mathrm{C} \square \rightarrow \mathrm{A})$. But, were $\mathrm{C}$ to obtain, it would not be the case that

\footnotetext{
${ }^{10}$ We don't need to add that $\mathrm{C}$ is compossible with $\mathrm{A}$, since that follows from C's possibility and A's necessity.

11 If $\mathrm{F}$ is contingent, then $\neg \mathrm{F}$ is a possible circumstance. Hence $\neg \mathrm{F}$ is in the set of all possible circumstances. However, $\neg \mathrm{F}$ is not in the set of all compossible-with-F-circumstances. And given that all the compossible-with-F-circumstances are also possible circumstances, it follows that the former is a proper subset of the latter.

12 Thanks to Marc Lange for suggesting this response.

13 Thanks to an anonymous referee for suggesting this response.
} 
F, which falsifies CE-N. Further, were C to obtain, then, since F does not obtain, F cannot be responsible for anything, let alone A. So TR-N is false too.

Strikingly, the counterexamples to $\mathbf{C E - N}$ and TR-N are cases where we have a contingent necessity-maker. This means that CE-N and TR-N are substantive assumptions, especially in the context of a debate about the (im)possibility of contingent necessity-makers. So Lange cannot simply assume them (otherwise, he is straightforwardly begging the question).

But it is also not obvious how Lange could motivate them. Recall that Lange argued we should accept CE and TR because they were borne out in his three examples. These examples offer no support to CE-N and TR-N, however: the examples all involve contingent, rather than necessary, A's, making them irrelevant to the truth or falsity of CE-N and TR-N. So nothing said so far makes a case for these two. And when we couple this lack of motivation with the fact that the two are substantive, then it is fair to say that employing them in this context is dialectically dodgy. Consequently, Lange could not adequately respond by restricting in this way either.

The upshot is that restricting the relevant principles does not in fact help Lange in his argument against contingent necessity-makers.

\section{Second Response: Rejecting the Counter-Examples}

All this might push Lange towards another, second line of response. The magnet reversal example turns upon the claim that $p$ 's being positively charged is responsible for the truth of the counterfactual that were $m$ to undergo magnetic reversal, $p$ would be negatively charged. One might object that it is not the case that $p$ 's being positively charged is responsible for the truth of the relevant counterfactualinstead, $p$ 's being positively charged is merely part of the necessary background conditions for the counterfactual's truth. Generalizing, we get the second response: the would-be counter-examples all mistakenly conflate necessary-condition-hood with responsibility.

However, this point equally applies to Lange's three test cases. According to Lange, what explains that match $m$ would have lit had it been struck is that $m$ is dry and oxygenated (and that various relevant laws of nature hold). But it seems that $m$ 's being dry and oxygenated is merely a necessary background condition for the truth of the counterfactual, not a genuine explanation (especially if we add the parenthetical clause about the laws of nature!). Similarly, that the field strength in $l$ is 1 dyne per statcoulomb is just a necessary background condition, rather than a proper explanation, because we also need to include all the other facts about the world that are relevant to $l$ (i.e. that a field-strength-changing particle didn't enter). Finally, Billy's accurately throwing his rock is only part of a complete explanation-we also need to add facts about e.g. the bottle's fragility, the rock's velocity/mass, that there are no bottle-protecting wizards around, etc. In short, if Lange wants to reject the counter-examples by saying that the cited F is only a necessary background condition, similar reasoning shows that his test cases should also be rejected.

That might not sound too bad, but remember that, without Lange's trio of cases, we've no reason to buy $\mathbf{C E}$. Recall that the first motivation was generalizing from 
the cases; clearly this won't do, since the cases turn out to incorrectly identify necessary background conditions as explanations. But we can also derive $\mathbf{C E}$ from TR and EF. However, the only reason Lange gave us for believing TR is that it was borne out in the cases. And if the cases turn out to be a poor guide, then there's nothing supporting TR, leaving CE without a leg to stand on. Consequently, if Lange responds by claiming that the counter-examples mistakenly equate a necessary background condition with an explanation, then his susceptibility to the same point leaves one of his key assumptions-and, more generally, his argument-unsupported.

\section{Conclusion}

The general consequence is that $\mathbf{C E}$ is either false or unmotivated. Either way, Lange's argument, which hinges upon $\mathbf{C E}$, is in significant trouble.

One upshot of this result is that the contingency horn of Blackburn's dilemma remains in principle graspable - a welcome result if one is interested in the overarching prospect of grounding modality in contingent matters. Of course, knocking down arguments against contingent necessity-makers is much different from offering a convincing positive case; that is work that still needs doing. ${ }^{14}$ Further, the present result only proves one argument against contingent necessity-makers fails; there are, however, others that must also be addressed if we are to offer such grounds for necessity. ${ }^{15}$ So, much more needs to be said if we want to fully address the question of whether contingencies can play the role of necessity-makers.

However, these are tasks for another day. For now, we can at least conclude that Lange has not yet given us a cogent reason to reject the thought that, at least in principle, contingencies can necessities make after all.

Acknowledgements Special thanks to Amanda Cawston and Michael Clark for extensive discussion, and for not letting me give up on this paper. Thanks also to Christian Folde, Marc Lange, Benjamin Schnieder, Robert Schwartzkopff, Richard Woodward, two anonymous referees for this journal, and audiences in Geneva, Helsinki, and Hamburg for helpful discussion. This paper was written partially under the auspices of the Singergia project Grounding: Metaphysics, Science, and Logic (Project 147685), funded by the Swiss National Sciences Foundation; I gratefully acknowledge their support.

Open Access This article is distributed under the terms of the Creative Commons Attribution 4.0 International License (http://creativecommons.org/licenses/by/4.0/), which permits unrestricted use, distribution, and reproduction in any medium, provided you give appropriate credit to the original author(s) and the source, provide a link to the Creative Commons license, and indicate if changes were made.

14 Though see Wildman (ms) for such an argument.

15 See e.g. Hanks (2008) and Hale (2013). 


\section{References}

Blackburn, S. (1986). Morals and modals. In G. Mcdonald \& C. Wright (Eds.), Fact, science, and morality: Essays on A.J. Ayer's language, truth, and logic (pp. 119-141). Oxford: Blackwell. Reprinted in Blackburn, S. (1993). Essays in quasi-realism (pp. 52-74). Oxford: Oxford University Press.

Cameron, R. (2010). On the source of necessity. In B. Hale \& A. Hoffman (Eds.), Modality (pp. 137153). Oxford: Oxford University Press.

Hale, B. (2002). The source of necessity. Nous, 36, 299-319.

Hale, B. (2013). Necessary beings: An essay on ontology, modality, and the relations between them. Oxford: Oxford University Press.

Hanks, P. (2008). A dilemma about necessity. Erkenntnis, 68, 129-148.

Kment, B. (2006). Counterfactuals and the analysis of necessity. Philosophical Perspectives, 20, 237-302.

Lange, M. (2008). Why contingent facts cannot necessities make. Analysis, 68, 120-128.

Lewis, D. (1973). Counterfactuals. Oxford: Blackwell.

Morato, V. (2014). Explanation and modality: On the contingency horn of Blackburn's dilemma. Erkenntnis, 79, 327-349.

Wildman, N. (2017). A note on Morato on modality and explanation. Erkenntnis, 82, 967-974.

Wildman, N. (ms). Necessity by accident. Unpublished manuscript.

Williamson, T. (2007). The philosophy of philosophy. Oxford: Oxford University Press.

Publisher's Note Springer Nature remains neutral with regard to jurisdictional claims in published maps and institutional affiliations. 